\title{
MEMS BASED BRIDGE MONITORING SUPPORTED BY IMAGE-ASSISTED TOTAL STATION
}

\author{
M. Omidalizarandi ${ }^{1, *}$, I. Neumann ${ }^{1}$, E. Kemkes ${ }^{2}$, B. Kargoll ${ }^{3}$, D. Diener ${ }^{1}$, J. Rüffer ${ }^{2}$, J.-A. Paffenholz ${ }^{1}$ \\ ${ }^{1}$ Geodetic Institute, Leibniz University Hannover, Nienburger Str.1, 30167 Hannover, Germany - (zarandi, neumann, diener, \\ paffenholz)@gih.uni-hannover.de \\ ${ }^{2}$ Allsat GmbH, 30165 Sokelant Str.5, Hannover, Germany - (eva.kemkes, juergen.rueffer@allsat.de) \\ ${ }^{3}$ Institut für Geoinformation und Vermessung Dessau, Hochschule Anhalt, Bauhaus Str. 8, 06846 Dessau-Roßlau, Germany - \\ (boris.kargoll@hs-anhalt.de)
}

KEY WORDS: Displacement and vibration analysis, MEMS accelerometer, Image-assisted total station, Modal parameter identification, Robust parameter estimation, Kalman filter, Bridge monitoring

\begin{abstract}
:
In this study, the feasibility of Micro-Electro-Mechanical System (MEMS) accelerometers and an image-assisted total station (IATS) for short- and long-term deformation monitoring of bridge structures is investigated. The MEMS sensors of type BNO055 from Bosch as part of a geo-sensor network are mounted at different positions of the bridge structure. In order to degrade the impact of systematic errors on the acceleration measurements, the deterministic calibration parameters are determined for fixed positions using a KUKA youBot in a climate chamber over certain temperature ranges. The measured acceleration data, with a sampling frequency of $100 \mathrm{~Hz}$, yields accurate estimates of the modal parameters over short time intervals but suffer from accuracy degradation for absolute position estimates with time. To overcome this problem, video frames of a passive target, attached in the vicinity of one of the MEMS sensors, are captured from an embedded on-axis telescope camera of the IATS of type Leica Nova MS50 MultiStation with a practical sampling frequency of $10 \mathrm{~Hz}$. To identify the modal parameters such as eigenfrequencies and modal damping for both acceleration and displacement time series, a damped harmonic oscillation model is employed together with an autoregressive (AR) model of coloured measurement noise. The AR model is solved by means of a generalized expectation maximization (GEM) algorithm. Subsequently, the estimated model parameters from the IATS are used for coordinate updates of the MEMS sensor within a Kalman filter approach. The experiment was performed for a synthetic bridge and the analysis shows an accuracy level of sub-millimetre for amplitudes and much better than $0.1 \mathrm{~Hz}$ for the frequencies.
\end{abstract}

\section{INTRODUCTION}

\subsection{Motivation}

For more than 10 years now, the damage to road bridges from the $60 \mathrm{~s}$ and $70 \mathrm{~s}$, which has been increasingly noticed, has been attracting more and more attention from the media. Besides this, the monitoring of bridges with different tasks and methods came into the focus of attention. These questions require an interdisciplinary cooperation between geodesists, civil engineers and geotechnical engineers.

Over the last decade of bridge monitoring, the authors and most likely other researchers as well as infrastructure operators have made different experiences. Initially, the scepticism towards permanent monitoring with geodetic sensors and methods was particularly high, but this methodology has gained an increasing reputation due to positive practical examples on selected bridges and other structures such as locks and dams. The impressive demonstration of geodetic methods soon led to the use of this technology in several cases: (1) for extensive rehabilitation measures on existing bridges, (2) to prevent early decommissioning, and (3) to ensure sufficient safety for people and the structure itself during operation. In combination with geotechnical monitoring procedures, this leads to undeniable economic advantages for the operators and for the society.

However, the infrastructure operators, in particular the road construction authorities in Germany, were soon confronted with the demand for a massive expansion of the geomonitoring of bridges. Not only in the case of extensive rehabilitation measures, but also for the preventive monitoring of bridges with condition grades 3 according to DIN 1076. In particular, a high percentage of $21 \%$ of all bridges built before 1980 in Germany was affected.

In particular, the high initial investment in the permanent use of geodetic methods caused the initial interest of the operators to drop considerably. The reason was seen in an economically unacceptable permanent monitoring of dilapidated, severely damaged or endangered bridges. And as long as nothing serious happened, the existing methodology for bridge monitoring seemed to be sufficient and in particular economically justifiable.

The increasing number of critical bridge structures with a simultaneous rehabilitation backlog and a lack of experienced experts for the inspection and assessment of bridges suggests the question of whether there are not effective and inexpensive methods that only initiate a visual inspection or precise geodetic monitoring of bridges when verifiable indications are available through efficient and cost-effective monitoring procedures. Then the targeted and detailed inspection and monitoring of structures classified as critical could be initiated as required.

The authors' goals are the self-sufficient use of a larger number of cost-effective and redundantly arranged sensors (here: MicroElectro-Mechanical System (MEMS) technology from the automotive industry) and the intelligent and continuous evaluation of data permanently obtained from potentially critical bridge structures based on frequency- and time-domain analysis methods. The following partial goals are in focus: (1) early and 
timely detection of variations of the modal parameters relevant for the structural condition of the bridge (e.g. natural frequencies and eigenforms), (2) permanent control and long-term statements for structural deformations and (3) allocation of deformations to the relevant influencing variables on the building deformation.

Therefore, this paper is organized as follows: The ongoing section gives a brief literature overview. Section 2 introduces the sensor specifications, the measurement set-up and the data acquisition. In Section 3 the suitability analysis of triaxial MEMS accelerometers for the purpose of bridge monitoring is discussed. The robust and automatic identification of modal parameters is introduced in Section 4. Section 5 presents the fusion of MEMS and image-assisted total station (IATS) data for a displacement analysis based on Kalman filtering. The realised experiment in Section 6 is sketched for a synthetic bridge, which is constructed by the Bundesanstalt für Materialforschung und -prüfung (BAM). The results of the experiment for the BAM synthetic bridge using the MEMS and the IATS are discussed in Section 7. The conclusion and the outlook on future work is given in Section 8 .

\subsection{Literature review}

For the monitoring of long-term movements of bridges (induced by foundation settlement, creep, and stress relaxation) or shortterm movements (caused by wind and traffic) (Duff and Hyzak, 1997), different contact-based or non-contact-based measurement methods can be utilised. As a surveying engineer's point of view, typically geodetic measurement systems, such as the global navigation satellite system (GNSS), robotic total stations (RTS), IATS, terrestrial laser scanner (TLS), laser tracker and/or other sensors such as accelerometers or digital cameras, can be used in the context of vibration monitoring of bridge structures. In the following, a brief and exemplarily overview of the state of the art based on a literature review is given. Roberts et al. (2004) utilised the hybrid measurement system consisting of a GNSS with a sampling frequency of $10 \mathrm{~Hz}$ and a triaxial accelerometer with sampling frequency of $200 \mathrm{~Hz}$ for a bridge deflection monitoring. Both measurement systems were complementary to each other since the GNSS measurements were used to suppress the accumulation drift of the acceleration data over time through zero velocity updates (ZUPT) and coordinate updates (CUPT). In order to filter out the high frequency noise, static test measurements with the accelerometers were carried out to identify those spurious frequencies induced by the sensor noise. Subsequently, the designed low-pass or band-pass filters were defined to suppress those frequencies with respect to the structural specifications and the results of the aforementioned static test. This is a challenging issue while the natural frequencies of bridges are located in the same band as those spurious frequencies. In addition, the hybrid measurement suffers from the drawback that the accuracy of the GNSS measurements are degraded due to multipath and cycle slips errors or poor satellite coverage. On the other hand, the state-of-the-art GNSS measurements cannot detect submillimetre displacement changes induced by higher natural frequencies. Neitzel et al. (2012) performed a vibration analysis of a bridge for a single point located along the bridge using a sensor network of accelerometers with a sampling frequency of $600 \mathrm{~Hz}$, a TLS (Zoller+Fröhlich Imager 5003) with a sampling frequency of $7812 \mathrm{~Hz}$ in single-point measurement mode and a terrestrial interferometric synthetic aperture radar (t-InSAR) with a sampling frequency of $200 \mathrm{~Hz}$ for a validation. In order to overcome the deficiency of the TLS in detecting such small displacements in the submillimetre range, an averaging over 100 measurements was carried out to reach a practical sampling frequency of $78.12 \mathrm{~Hz}$. Psimoulis and Stiros (2013) used the RTS with non-constant sampling rate measurements in a range of 5$7 \mathrm{~Hz}$ for vibration monitoring of a short span railway bridge. Ehrhart and Lienhart (2015a) performed displacement and vibration monitoring of a footbridge structure by capturing video frames from the telescope camera of an IATS for a circular target marking rigidly attached to the structure. Ehrhart and Lienhart (2015b) and Lienhart et al. (2017) were carried out vibration analysis of a footbridge structure using an IATS of type Leica MS50 with a sampling frequency of $10 \mathrm{~Hz}$, an RTS of type Leica TS15 with a sampling frequency of $20 \mathrm{~Hz}$ and an accelerometer of type HBM B12/200 with a sampling frequency of $200 \mathrm{~Hz}$ based on measurements of the circular target markings and structural features such as bolts of the bridge structure. Omidalizarandi et al. (2018) used an IATS of type Leica MS50 with sampling frequency of $10 \mathrm{~Hz}$ for displacement and vibration analysis of a footbridge structure. Schill and Eichhorn (2019) employed a phase-based profile scanner of type Zoller+Fröhlich Profiler 9012 with a sampling frequency of $50 \mathrm{~Hz}$ for deformation monitoring of the bridge structures.

\section{SENSOR SPECIFICATIONS, MEASUREMENT SET- UP AND DATA ACQUISITION}

The MEMS sensors of type BNO055 from the Bosch company have been used in this study. It includes a triaxial 14-bit accelerometer, a triaxial 16-bit gyroscope and a triaxial magnetometer, which allows to acquire acceleration, rotation and magnetic field strength, respectively (see manufacturer's data sheet for details in Bosch (2016)). However, we merely benefit its accelerometer sensor to record the acceleration data in three main directions of a bridge structure (i.e. longitudinal, lateral and vertical directions). Its maximum sampling frequency is $200 \mathrm{~Hz}$. But, we set it to $100 \mathrm{~Hz}$ by considering typical eigenfrequency ranges of the bridge structures between 0.1 up to minimum $25 \mathrm{~Hz}$, which is sufficient in the light of Nyquist-Shannon sampling theorem. Acceleration ranges can be defined in the ranges of $\pm 2 g / \pm 4 g / \pm 8 g / \pm 16 g$, which is here set to $\pm 2 g$. At the moment, a geo-sensor network of the MEMS accelerometers includes a master node and three measuring nodes that are so called master and slaves, respectively.

The measurement starts by triggering the slave sensors from the master sensor via cable. The acceleration measurements are recorded into the SD memory card in blocks. Each memory block has a time stamp transmitted by the master, which is provided by an integrated low-cost GNSS equipment. Both, the master and the slaves contain a Bosch BNO055 accelerometer and a control/storage unit. The components are mounted in an aluminium housing, which protects the electronics against temperature and humidity changes, wind and rain by means of a suitable design.

To perform evaluation and validation of the estimated modal parameters from the MEMS accelerometers, a highly accurate reference sensor is employed. We have used a laser tracker of type Leica AT960-LR with a maximum permissible error of $15 \mu \mathrm{m}+6 \mu \mathrm{m} / \mathrm{m}$ for a 3D point with a sampling frequency of $1000 \mathrm{~Hz}$ (Hexagon Metrology, 2015). Therefore, the use of the laser tracker as a reference sensor allows high measuring accuracy with a high and precise measuring frequency.

An IATS of type Leica Nova MS50 MultiStation is utilized to perform 1D CUPT of the acceleration data acquired by the MEMS accelerometers in the vertical direction. The angular accuracy is $1 "$ according to ISO 17123-3. The outputs of the IATS are polar measurements, which can be used to calculate 
Cartesian coordinates. Such 3D coordinates of the measured object are not practically suitable to perform displacement analysis in a sub-millimetre range. To overcome this problem, a $1 \mathrm{D}$ displacement time series is generated based on continuous extraction of the passive target centroids from the video frames captured from the embedded on-axis telescope camera of the IATS with practical sampling frequency of $10 \mathrm{~Hz}$. The conversion of the $1 \mathrm{D}$ displacement changes from the pixel unit to the metric unit is carried out based on a vertical angular conversion factor, target object size as well as a slope distance measured to the aforementioned passive target. For more information regarding the calibration procedure of the vertical angular conversion factor, please refer to cf. Ehrhart and Lienhart (2015a) or Omidalizarandi et al. (2018). It should be noted that merely $1 \mathrm{D}$ displacement time series has been generated using the aforementioned video frames due to availability of only one IATS at time of the measurements, which subsequently allows to perform just 1D CUPT in this study.

The passive target centroids are extracted based on the work of Omidalizarandi et al. (2019a). The used target pattern comprises a circular border with four intersected line patterns (Figure 1). The procedure starts by manual initial sighting to the target at the beginning of the measurements and measuring its corresponding slope distance. Next, the images obtained from captured video frames are cropped based on a target object size as well as the slope distance. The median blur and bilateral filtering are applied for the purpose of noise reduction and sharp edge preservation. The line segment detector (LSD) (Grompone von Gioi et al., 2012) is applied to extract line features. A histogram of azimuth of the extracted lines is calculated to select the lines with maximum azimuth bin values in an iterative procedure. Afterwards, the RANSAC algorithm is applied to those LSD lines with maximum deviation less than $15^{\circ}$ from the selected lines from the previous step. A Huber-robust line fitting (Kaehler and Bradski, 2016) is then applied to those LSD lines within a predefined buffer width around the RANSAC lines to increase the robustness and reliability of the extracted lines. To this end, the extracted lines are intersected and then clustered based on their proximity by considering a threshold of 2 pixels. At the end, the maximum cluster is selected and its weighted average value results in the final intersection point. For further details the reader is referred to Omidalizarandi et al. (2019a).

Figure 1 depicts the passive target attached to a frontal side of the BAM synthetic bridge in the vicinity of one the MEMS accelerometers, which is mounted at the bottom of the aforementioned synthetic bridge. In addition, a corner cube reflector is mounted to be measured by the laser tracker.

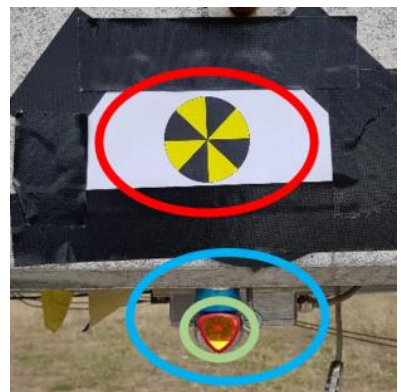

Figure 1. A passive target (located inside the red ellipse) attached to the BAM synthetic bridge, which is close to the MEMS (located inside the cyan ellipse) and corner cube reflector (located inside the green ellipse).

\section{SUITABILITY ANALYSIS OF TRIAXIAL MEMS ACCELEROMETERS}

The acceleration measurements recorded from the MEMS accelerometer yields accurate results over short time intervals but may suffer from accuracy degradation with time in particular for absolute position estimates calculated from the double integration. Therefore, due to combined effects of MEMS related systematic and random errors such as noises, biases, drifts and scale factor instabilities on its long-term measurements, a calibration procedure is carried out to compensate the systematic errors and to provide reliable measurement results.

To select a proper and optimal MEMS sensor despite of considering purchase price of the sensor and their sampling frequency, two scenarios are proposed. Firstly, a robust and reliable calibration procedure is developed and implemented to estimate the calibration parameters including three biases, three scale factors and three non-orthogonality angles between the axes in an automated manner. For this purpose, the calibration functions are defined based on common six-position static acceleration tests (cf. Shin and El-Sheimy, 2002) using a KUKA youBot (Figure 2). The used youBot enables us to perform calibration automatically for fixed positions and for certain time intervals. To have a better realisation of changes of the calibration parameters, the calibration procedure is carried out in a climate chamber over different temperature ranges between $10^{\circ} \mathrm{C}$ to $30^{\circ} \mathrm{C}$. Higher or lower temperatures were not possible due to climate chamber and youBot restrictions. To this end, such a calibration procedure allows to avoid in-situ calibration by estimating the calibration parameters based on the interpolation of the parameters estimated at different temperatures in the controlled environmental experiment. Due to the use of only $1 \mathrm{D}$ acceleration data in this study, the calibration of the nonorthogonality angles between the three axes may not play an important role. However, in our future research the 3D acceleration data in all three directions can be considered. Further explanation regarding the developed robust calibration procedure is also part of our future publication and it is out of scope of this study.

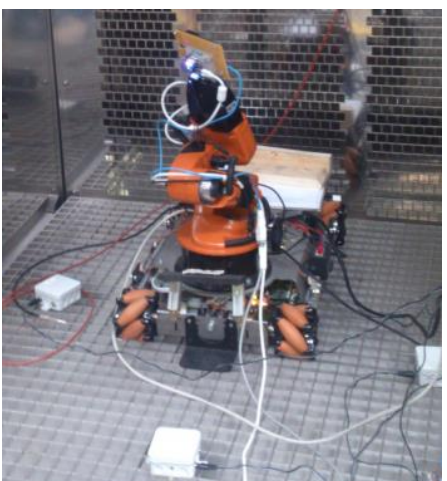

Figure 2. A calibration experiment using a KUKA youBot inside the climate chamber at the Institute of Thermodynamics, Leibniz University Hannover.

Secondly, controlled excitation experiments are conducted at the laboratory environment using a high-precision shaker (Figure 3). The advantages of such experiment are twofold: (1) it allows us to estimate harmonic oscillation parameters such as frequency, amplitude, damping ratio coefficient as well as phase shift and to compare them with those estimated parameters either from other slave MEMS accelerometers or from the reference sensors such as reference accelerometers or laser tracker. (2) The time 
synchronisation between MEMS accelerometers can be compared together based on the estimated phase shifts.

The measurements were performed at frequencies of 5, 10 and $20 \mathrm{~Hz}$. The shaker includes a highly accurate reference accelerometer recording the acceleration measurements with a sampling rate of $1024 \mathrm{~Hz}$ for the duration of 5 minutes.

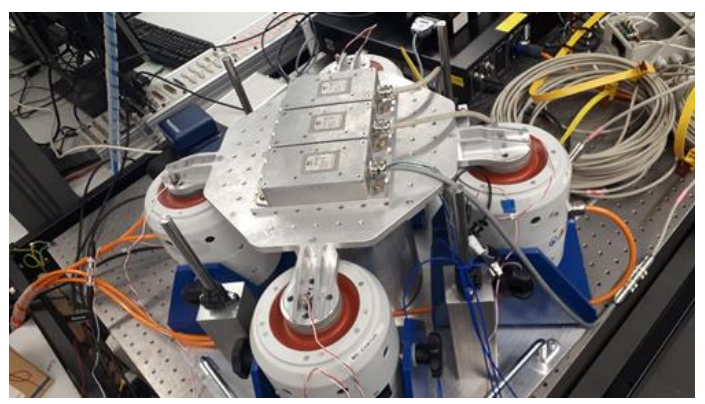

Figure 3. A controlled excitation experiment at the Institute of Dynamics and Vibration Research, Leibniz University

Hannover to validate the time synchronisation between all three slave MEMS accelerometers.

\begin{tabular}{|c|c|c|c|c|c|}
\hline $\begin{array}{c}\mathrm{f} \\
(\mathrm{Hz})\end{array}$ & $\begin{array}{c}\mathrm{A} \\
\left(\mathrm{m} / \mathrm{s}^{2}\right)\end{array}$ & $\begin{array}{c}\mathrm{A} \\
(\mathrm{mm})\end{array}$ & $\begin{array}{c}\varphi \\
\left({ }^{\circ}\right)\end{array}$ & $\begin{array}{c}|g| \\
\left(\mathrm{m} / \mathrm{s}^{2}\right)\end{array}$ & $p$ \\
\hline 5.0027 & 0.3449 & 0.3491 & 5.7263 & 9.82 & 71 \\
\hline 10.0054 & 1.5612 & 0.3950 & 153.204 & 9.82 & 88 \\
\hline 20.0109 & 5.8159 & 0.3679 & 101.052 & 9.88 & 81 \\
\hline $\begin{array}{c}\mathrm{f} \\
(\mathrm{Hz})\end{array}$ & $\begin{array}{c}\mathrm{A} \\
\left(\mathrm{m} / \mathrm{s}^{2}\right)\end{array}$ & $\begin{array}{c}\mathrm{A} \\
(\mathrm{mm})\end{array}$ & $\begin{array}{c}\varphi \\
\left.{ }^{\circ}\right)\end{array}$ & $\begin{array}{c}|\mathrm{g}| \\
\left(\mathrm{m} / \mathrm{s}^{2}\right)\end{array}$ & $p$ \\
\hline 5.0027 & 0.3512 & 0.3555 & 5.6675 & 10.42 & 71 \\
\hline 10.0054 & 1.5909 & 0.4025 & 153.147 & 10.42 & 88 \\
\hline 20.0109 & 5.9290 & 0.3751 & 100.859 & 10.53 & 81 \\
\hline
\end{tabular}

Table 1. Statistics of the controlled excitation experiment for a MEMS (IMU_slave_03) measurements with (top) and without (bottom) applying calibration parameters.

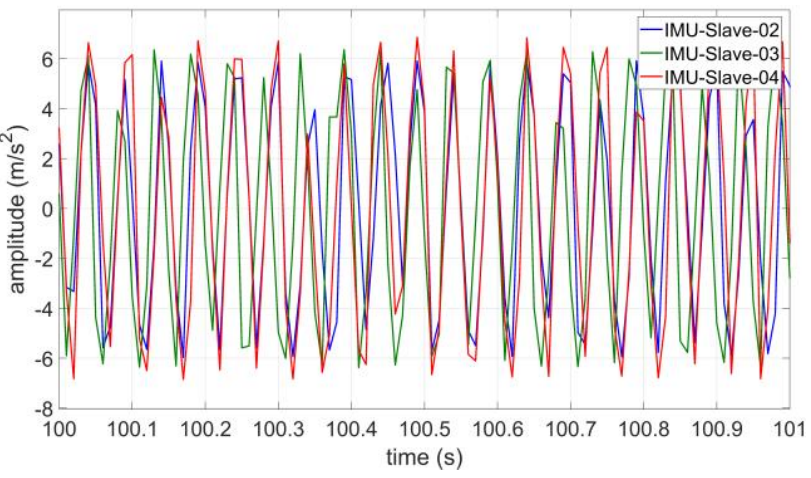

Figure 4. Overlaying of time series of the acceleration data recorded from all three slave MEMS sensors for a duration of

$1 \mathrm{~s}$ and at the frequency of $20 \mathrm{~Hz}$ within the controlled excitation experiment.

Figure 4 shows the overlaying of time series of the acceleration data recorded from all three slave MEMS sensors for a duration of $1 \mathrm{~s}$ and at the frequency of $20 \mathrm{~Hz}$. As we can see from this figure, the peaks of the measurements obtained from the two of the MEMS sensors (IMU_slave_02 and IMU_slave_04) have been better matched compared to another one (IMU_slave_03). However, the time delay between their measurements is approximately $0.01 \mathrm{~s}$, which is still acceptable for our application in this study.

The analysis of the second scenario is exemplarily provided in Table 1 for one slave MEMS accelerometer, namely, IMU_slave_03 in two cases: (1) with and (2) without applying the calibration parameters. The estimated parameters include the frequency $(\mathrm{Hz})$, the amplitudes in both units of $\left(\mathrm{m} / \mathrm{s}^{2}\right)$ and $(\mathrm{mm})$, the phase shift $\left({ }^{\circ}\right)$, the calculated absolute gravity value based on the calibration parameters $\left(\mathrm{m} / \mathrm{s}^{2}\right)$ and an autoregressive (AR) model order, for which the estimation procedure is described in Section 4. Further analyses show that the phase shifts between sensors vary between 2 to 5 degrees. Moreover, the differences of approximately $0.003 \mathrm{~Hz}$ for the frequency of $5 \mathrm{~Hz}$ and $0.01 \mathrm{~Hz}$ for the frequency of $20 \mathrm{~Hz}$ in comparison with the nominal frequency values are realised. Such differences prove that the time synchronisation has greater influence at higher frequencies. Additionally, the analyses prove a very less influence of the calibration parameters on the estimated modal parameters for a short period of time.

\section{ROBUST AND AUTOMATIC IDENTIFICATION OF MODAL PARAMETERS}

To inspect the changes in the global dynamic behaviour of the structure such as natural frequencies, mode shapes (i.e. eigenforms) and modal damping, a proper functional model should be identified. To tackle this problem and to estimate amplitudes as well as frequencies for displacement and vibration analysis, a linear regression model in terms of a sum of sinusoids and the AR model of the coloured measurement noise were previously employed and solved by means of the generalized expectation maximization (GEM) algorithm (Omidalizarandi et al. 2018). However, the estimated amplitude might be influenced by the damping characteristics of the structure, which the previous undamped model does not capture. Therefore, the deterministic model is extended to a damped harmonic oscillation (DHO) introduced in Amezquita-Sanchez and Adeli (2015) and applied by Kargoll et al. (2019) to estimate frequency, amplitude, phase shift as well as damping ratio coefficient.

We modelled the given acceleration measurements $l_{1}, \ldots, l_{n}$ according to the DHO model

$$
\begin{aligned}
l_{t}=\frac{a_{0}}{2} & +\sum_{j=1}^{M}\left[a_{j} \cos \left(2 \pi f_{j} \sqrt{1-\xi_{j}^{2}} x_{t}\right)\right. \\
& \left.+b_{j} \sin \left(2 \pi f_{j} \sqrt{1-\xi_{j}^{2}} x_{t}\right)\right] \\
& \times \exp \left(-2 \pi \xi_{j} f_{j} x_{t}\right)+e_{t}
\end{aligned}
$$

where the time instances $x_{1}, \ldots, x_{n}$ correspond to the ambient window of the acceleration time series and where the undamped frequencies $f_{1}, \ldots, f_{M}$, the coefficients $a_{0}, a_{1}, \ldots, a_{M}$, and $b_{1}, \ldots, b_{M}$ as well as damping ratio coefficients $\xi_{1}, \ldots, \xi_{M}$ are treated as unknown parameters. Subsequently, the damped frequencies are calculated by $f_{j d}=f_{j} \sqrt{1-\xi_{j}^{2}}$. It should be noted that the acceleration measurements are subtracted from their mean value for each selected ambient window.

To deal with the strong coloured measurement noise in the acceleration measurements, we assume the random deviations $\left(e_{t}\right)$ are autocorrelated through a covariance-stationary $\mathrm{AR}$ process as proposed by Kargoll et al. (2018) 


$$
e_{t}=\alpha_{1} e_{t-1}+\ldots+\alpha_{p} e_{t-p}+u_{t}
$$

in which the coefficients $\boldsymbol{\alpha}^{\boldsymbol{T}}=\left[\alpha_{1}, \ldots, \alpha_{p}\right]$ are also considered as unknown parameters. Since we expect numerous outliers of different magnitudes to be present in the data, the white noise components $u_{1}, \ldots, u_{n}$ of that AR noise model are assumed to follow the centred and scaled t-distribution $t_{v}\left(0, \sigma^{2}\right)$, where the degree of freedom $v$ and scale factor $\sigma^{2}$ are treated as further parameters to be estimated, as suggested by Kargoll et al. (2018).

Equation (1) only achieves reasonable results while the measurements do not have a significant drift. Therefore, acceleration measurements should be detrended by means of the calibration parameters to compensate a linear bias drift. However, this method is not applicable to the displacement time series obtained by using the IATS. Alternatively, the offset $\frac{a_{0}}{2}$ in Equation (1) can be replaced by $c_{0}+c_{1} x_{t}$ to account for the linear drift either of the acceleration or displacement measurements. This model can be made more complex for displacement time series with higher orders, which extensions are, however, beyond the scope of this study.

To estimate the model parameters by means of the aforementioned GEM algorithm, the number $M$ of Fourier frequencies and the model order $p$ of the AR process are specified beforehand. The required initial frequency values $f_{1}^{(0)}, \ldots, f_{M}^{(0)}$ and the AR model order are defined as explained in Omidalizarandi et al. (2019b). In addition, the initial damping ratio coefficients are set to 0 .

\section{FUSION OF MEMS AND IATS FOR DISPLACEMENT ANALYSIS BASED ON KALMAN- FILTERING}

Typically, the 3D coordinate measurements of a state of the art IATS are at the level of $1 \mathrm{~mm}$ accuracy or above depending on the distance to the object, which are not sufficiently accurate to characterise such small displacements at the level of submillimetre ranges for the application of bridge displacement monitoring. To achieve this goal in this study, the 1D displacement time series generated from the passive target centroid detection are fused with the acceleration measurements recorded from one of the MEMS accelerometers. Such a data fusion is also beneficial to overcome the 1D displacement drift of the MEMS accelerometer over time. For this purpose, the 1D CUPT is performed based on an iterative extended Kalman filter (iEKF). The Kalman filter is a recursive optimal filter which is carried out in three steps of initialisation, prediction and filtering. For more information in this regard, please refer to Kalman (1961).

The state vector $\boldsymbol{y}^{(\boldsymbol{k})}$ at epoch $\mathrm{k}$ is described as

$$
\boldsymbol{y}^{k}=\left[d^{k}, v^{k}, a^{k}\right]
$$

here $d^{k}, v^{k}, a^{k}$ are the displacements, the velocities and the accelerations at the epoch $\mathrm{k}$ in the vertical direction. Since the acceleration and displacement are acquired with different sampling frequencies of $100 \mathrm{~Hz}$ and $10 \mathrm{~Hz}$, respectively, the prediction step allows to compensate the displacement gaps. Alternatively, the displacement time series can be reconstructed from the estimated DHO model parameters to fill out those displacement gaps with resampling. The predicted state vector $\left(\overline{\boldsymbol{y}}^{k+1}\right)$ at epoch $\mathrm{k}+1$ is calculated based on the linear system equation as

$$
\overline{\boldsymbol{y}}^{k+1}=\boldsymbol{\Phi}^{k} \cdot \widehat{\boldsymbol{y}}^{k}+\boldsymbol{G}^{k} \cdot \boldsymbol{w}^{k}+\boldsymbol{L}^{k} \cdot \boldsymbol{u}^{k}
$$

where $\boldsymbol{\Phi}^{k}$ is the transition matrix calculated from the dynamic model of the system (i.e. equations of motion) with respect to the state vector parameters, $\widehat{\boldsymbol{y}}^{k}$ is the updated state vector at epoch $\mathrm{k}, \boldsymbol{G}^{k}$ and $\boldsymbol{w}^{k}$ are the matrix and vector of disturbing variables or noises, $\boldsymbol{L}^{k}$ and $\boldsymbol{u}^{k}$ are the matrix and vector of acting forces. It should be noted that the influence of acting forces is neglected to simplify the problem.

The observation model is determined as

$$
\begin{aligned}
\boldsymbol{l}^{k+1} & =\mathbf{A}^{k+1} \cdot \widehat{\boldsymbol{y}}^{k+1}+\boldsymbol{v}^{k+1}, \\
{\left[\begin{array}{l}
d^{k+1} \\
a^{k+1}
\end{array}\right] } & =\left[\begin{array}{lll}
1 & 0 & 0 \\
0 & 0 & 1
\end{array}\right]\left[\begin{array}{l}
d^{k+1} \\
v^{k+1} \\
a^{k+1}
\end{array}\right]+\boldsymbol{v}^{k+1}
\end{aligned}
$$

where $\boldsymbol{l}$ is the observation vector, $\mathbf{A}$ is the design matrix and $\boldsymbol{v}$ is the vector of residuals. The transition matrix is then given by integration with respect to the state vector parameters as

$$
\boldsymbol{\Phi}^{k}=\left[\begin{array}{ccc}
1 & \Delta t & \frac{1}{2} \Delta t^{2} \\
0 & 1 & \Delta t \\
0 & 0 & 1
\end{array}\right]
$$

where $\Delta t$ is the sampling period. The covariance matrix of the process noise is defined based on Wiener-sequence acceleration as described by Bar-Shalom et al. (2001)

$$
\boldsymbol{\Sigma}_{\boldsymbol{w w}}=\left[\begin{array}{ccc}
\frac{1}{20} \Delta t^{5} & \frac{1}{8} \Delta t^{4} & \frac{1}{6} \Delta t^{3} \\
\frac{1}{8} \Delta t^{4} & \frac{1}{3} \Delta t^{3} & \frac{1}{2} \Delta t^{2} \\
\frac{1}{6} \Delta t^{3} & \frac{1}{2} \Delta t^{2} & \Delta t
\end{array}\right] \tilde{q}
$$

where $\tilde{q}$ is the ratio between the system noise and observation noise. The covariance matrix of the observations is given by

$$
\boldsymbol{\Sigma}_{l l}=\left[\begin{array}{cc}
\sigma_{d}^{2} & 0 \\
0 & \sigma_{a}^{2}
\end{array}\right]
$$

where $\sigma_{d}$ and $\sigma_{a}$ are the a-priori standard deviations of the displacements and the accelerations. It should be noted that the noise behaviour of the MEMS acceleration data at rest and for its 3 -axes (i.e. X, Y and Z) are approximately about $0.016,0.018$ and $0.045 \mathrm{~m} / \mathrm{s}^{2}$, respectively. However, the uncertainty for its $\mathrm{Z}$ axis at motion (i.e. under oscillation imposed by the modal hammer) is about $0.013 \mathrm{~m} / \mathrm{s}^{2}$ according to the analysis performed for the synthetic bridge described in Section 6. Subsequently, the apriori standard deviations are set to $0.0001 \mathrm{~m}$ and $0.013 \mathrm{~m} / \mathrm{s}^{2}$, respectively. For further details concerning the iEKF steps, please refer to cf. Omidalizarandi and Zhou (2013).

\section{EXPERIMENTAL SET-UP}

A kinematic deformation analysis of the BAM synthetic bridge structure, located in approximately $80 \mathrm{~km}$ distance from Berlin, Germany, with a length of $24.0 \mathrm{~m}$, a width of $0.8 \mathrm{~m}$ and a height of $0.3 \mathrm{~m}$ (Figure 5) is investigated. The measurements were carried out using the MEMS accelerometers, the laser tracker Leica AT960-LR and the IATS (Leica MS50) with sampling frequencies of $100 \mathrm{~Hz}, 1000 \mathrm{~Hz}$ and $10 \mathrm{~Hz}$, respectively.

To identify all possible mode shapes of the aforementioned BAM synthetic bridge, the three slave and one master MEMS 
accelerometers were attached at certain positions and at the first half of the synthetic bridge due to the symmetric behaviour of the mode shapes. Therefore, the three slave MEMS accelerometers are placed at the first, second and third quarters of the synthetic bridge. The master MEMS accelerometer is placed at the first quarter.

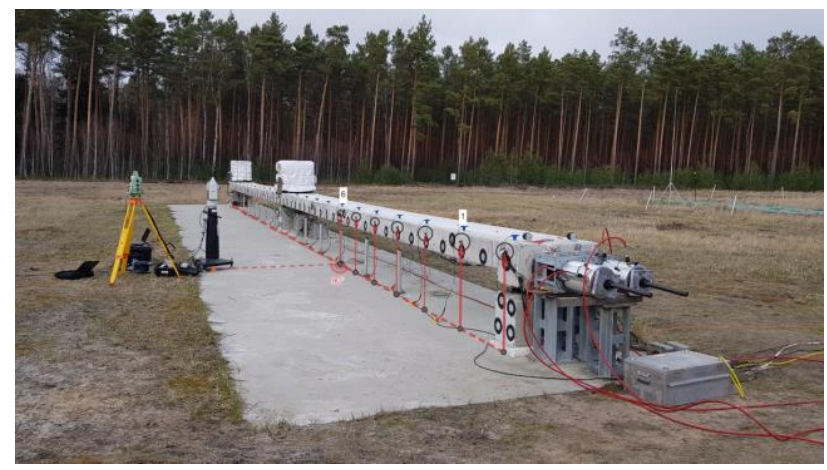

Figure 5. The BAM synthetic bridge measured by the MEMS accelerometers, the laser tracker and the IATS.

Since the BAM synthetic bridge is a rigid platform without any natural frequencies, therefore an artificial oscillation was generated using a modal hammer.

As mentioned before, the acceleration measurements were acquired from all three slave MEMS accelerometers in three directions. However, only the acceleration measurements in the $\mathrm{Z}$ direction were considered.

\section{RESULTS FOR THE MEMS ACCELEROMETERS AND THE IATS}

Figure 6 depicts the displacement time series obtained from the video frames captured by the telescope camera of the IATS at a centre position of the BAM synthetic bridge for a duration of 10 minutes. The large peaks illustrate the time instances while the external forces were imposed by the modal hammer. A steep rise at approximately $310 \mathrm{~s}$ is due to the bridge movement as it was visible from displacement time series obtained from the IATS and the laser tracker as well.

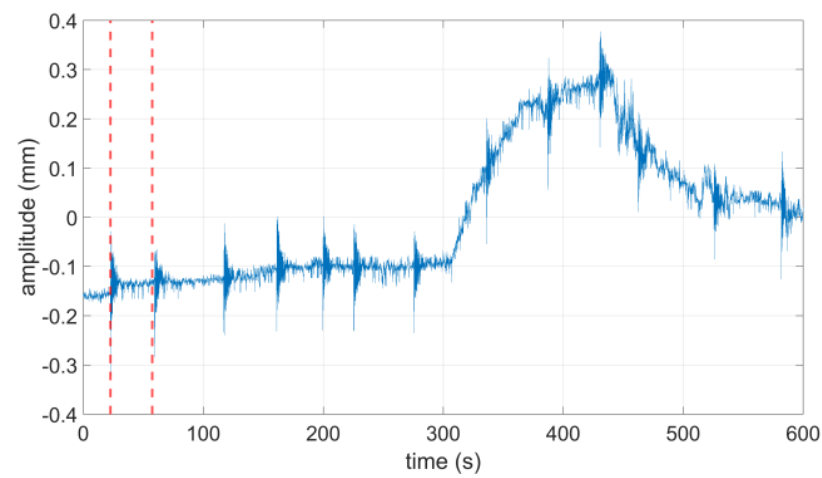

Figure 6. Displacement time series at the centre position of the BAM synthetic bridge, which was obtained from the video frames captured by the telescope camera of the IATS (blue solid), and selected ambient window of $35 \mathrm{~s}$ (red dashed lines).

Figure 7 shows the discrete Fourier transform (DFT) of the MEMS acceleration data. As we can see in this figure, it is very challenging to directly extract dominant eigenfrequencies from the DFT due to numerous spurious frequencies.
Figure 8 illustrates the time series of the 1D displacements from the IATS and its adjusted data within the selected ambient window. Figures (9-10) depict the eigenfrequencies estimated for the acceleration data from the MEMS and for the displacement data from the IATS. As we can see in Figure 9, there might be a correlation between closely spaced frequencies of 5.73 and 5.33 $\mathrm{Hz}$, which have amplitudes approximately close to the noise level of the acceleration data.

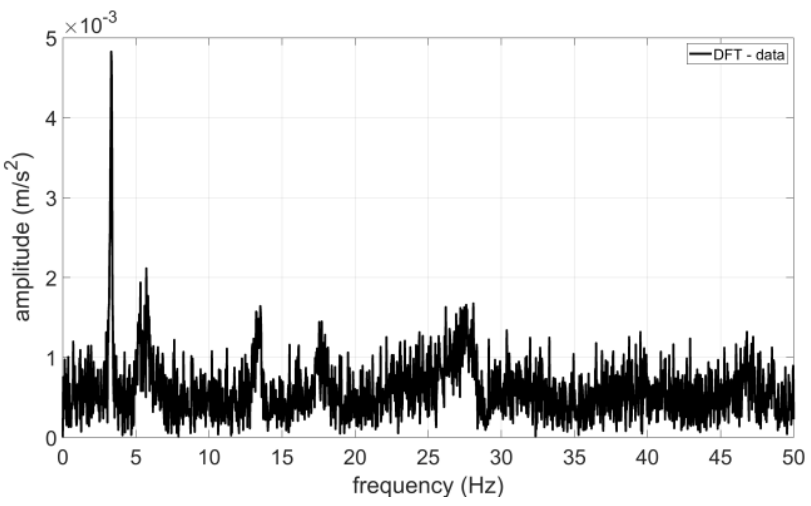

Figure 7. The DFT of the MEMS accelerations for the selected ambient window of $35 \mathrm{~s}$ shown in Figure 6.

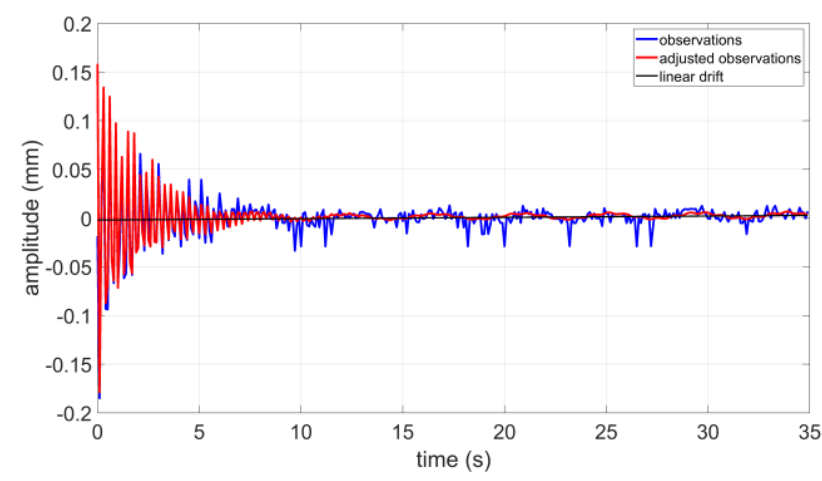

Figure 8. The time series of the displacements from the IATS within the selected ambient window (blue) and their adjusted observations (red).

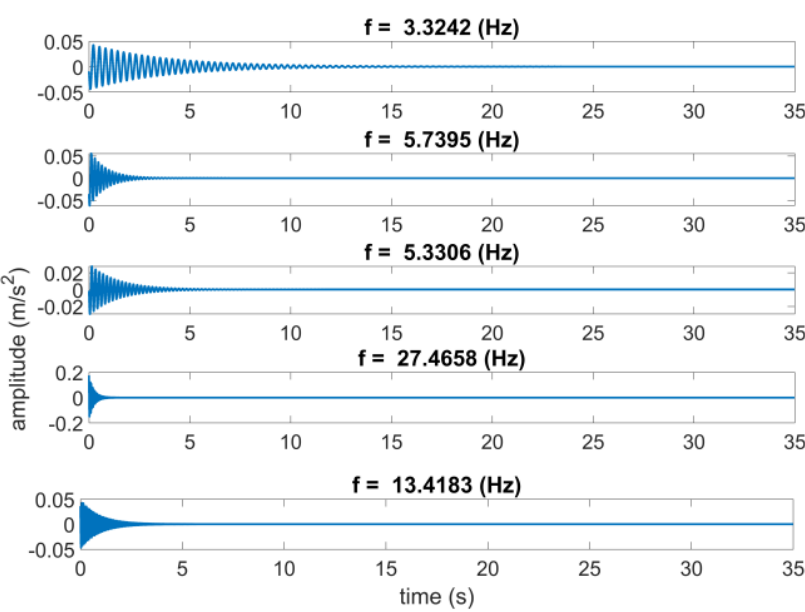

Figure 9. Identified frequencies from the MEMS acceleration data within the selected ambient window. 


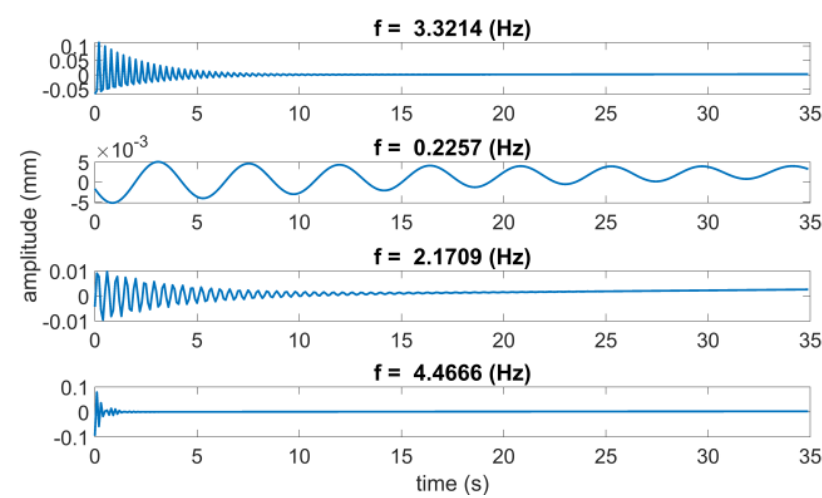

Figure 10. Identified frequencies for the displacement data from the IATS within the selected ambient window by considering the linear drift of the offset.

In real applications of bridge structures, the natural frequencies might be changed but may not be diminished, which can be seen for the frequency of $3.3 \mathrm{~Hz}$. In contrast, a system frequency caused by the imposition of the load will be diminished in a few seconds such as for the frequency of $27.46 \mathrm{~Hz}$ extracted from the acceleration data. The impact of the linear drift offset for the displacement data obtained from the IATS is visible for the lower frequency of $0.22 \mathrm{~Hz}$. The frequency of $4.46 \mathrm{~Hz}$ for the displacement data from the IATS shows a superimposition of higher frequencies than $5 \mathrm{~Hz}$, which is related to the deficiency of the IATS and its low sampling frequency.

In order to obtain the acceleration measurements at the metric unit, a double integration is performed within the selected ambient window based on the work of Omidalizarandi et al. (2019b). Subsequently, the displacements are estimated for each identified eigenfrequency by fitting the DHO model to the double integrated adjusted acceleration data (Figure 11).

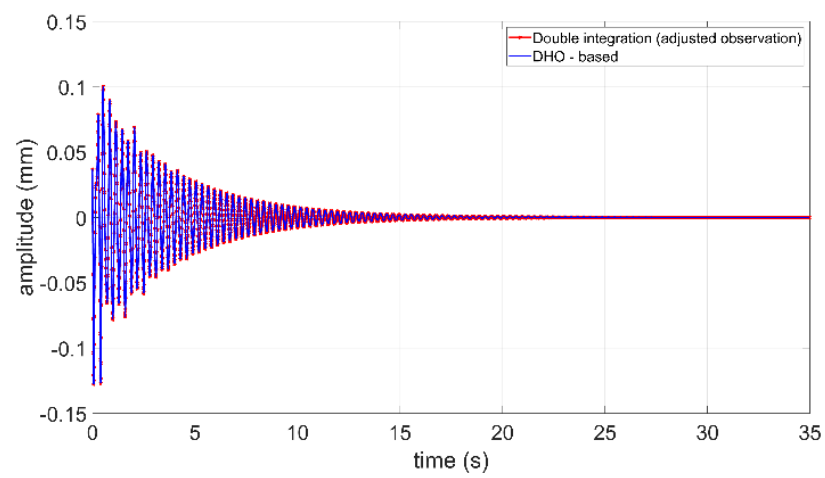

Figure 11. The calculated displacements for the adjusted MEMS acceleration data within the selected ambient window based on: double integration (red) and fitted DHO model (blue).

An eigenfrequency of $3.3 \mathrm{~Hz}$ is detected precisely from the measurements of the MEMS, the IATS and the laser tracker as we can see in Table 2 . The double integration accuracy might be degraded for the calculated displacements obtained from the MEMS acceleration data for a duration of $10 \mathrm{~s}$ or less in case of high load impact on the structure. This is due to the fact that the modal parameters might not be identified reliably for such short time interval. Furthermore, the analysis shows that the estimated damping ratio coefficients from the IATS and the laser tracker are not as accurate as from the MEMS due to superimposition of those higher frequencies with small amplitudes. The reason lies in the uncertainty of the measurements, which are higher than the amplitudes of the oscillations.

\begin{tabular}{|c|c|c|c|}
\hline Time: $15 \mathrm{~s}$ & $\begin{array}{c}\mathrm{f} \\
(\mathrm{Hz})\end{array}$ & $\begin{array}{c}\mathrm{A} \\
(\mathrm{mm})\end{array}$ & $\begin{array}{c}\xi \\
(\%)\end{array}$ \\
\hline MEMS & 3.3278 & 0.1064 & 1.30 \\
\hline IATS & 3.2968 & 0.1357 & 2.27 \\
\hline Laser tracker & 3.3190 & 0.1263 & 1.61 \\
\hline Time: $35 \mathrm{~s}$ & $\begin{array}{c}\mathrm{f} \\
(\mathrm{Hz})\end{array}$ & $\begin{array}{c}\mathrm{A} \\
(\mathrm{mm})\end{array}$ & $\begin{array}{c}\xi \\
(\%)\end{array}$ \\
\hline MEMS & 3.3242 & 0.0977 & 1.20 \\
\hline IATS & 3.3214 & 0.1217 & 1.97 \\
\hline Laser tracker & 3.3181 & 0.1282 & 1.64 \\
\hline
\end{tabular}

Table 2. Statistics of estimated modal parameters for the MEMS accelerometer, the IATS and the laser tracker measurements for a duration of $15 \mathrm{~s}$ (top) and $35 \mathrm{~s}$ (bottom) within the selected ambient window and at the centre position of the BAM synthetic bridge.

In order to perform a data fusion of different measurements obtained from different sensors, a time synchronisation between the sensors plays an important role. In an ideal case, the time synchronisation should be performed at the hardware level. However, in this study, as described in Ferrari et al. (2016), to obtain better synchronisation between the measurements and to calculate a time delay between them, a cross-correlation is carried out. But, this is a challenging issue since the outputs of the sensor measurements are in different units. To tackle this problem, the adjusted MEMS acceleration data are converted to a displacement vector at the metric unit based on a double integration for a duration of $35 \mathrm{~s}$ within the selected ambient window. Next, the adjusted MEMS acceleration data and the IATS displacement data are resampled to the sampling frequency of the laser tracker. Then the cross-correlation between the IATS and MEMS with respect to the laser tracker is calculated. The analysis shows that a maximum cross-correlation between the MEMS and the laser tracker (10.909) is slightly higher than the maximum cross-correlation between the IATS and the laser tracker (10.631) (see Fig. 12). This makes sense due to extraction of higher eigenfrequencies from the MEMS compared to the IATS.
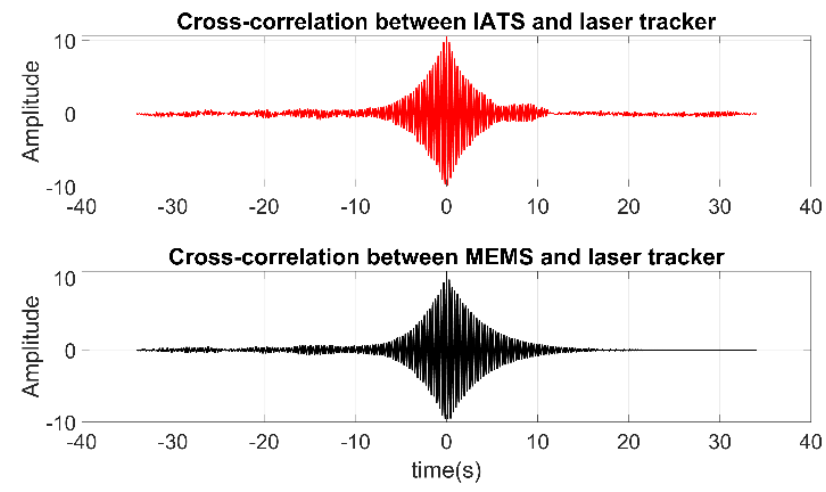

Figure 12. Cross-correlation between the displacement time series obtained from the IATS data, the double integration of the adjusted MEMS acceleration data and the laser tracker data

for a duration of $35 \mathrm{~s}$ within the selected ambient window.

Figure 13 depicts an overlay of the displacement time series from aforementioned sensors after performing the time synchronisation. It shows how the peaks nicely fit together. Additionally, as we can also see from this figure, the amplitudes 
of the peaks in the laser tracker and the MEMS are slightly higher than the IATS, which is due to the extracting the higher eigenfrequencies.

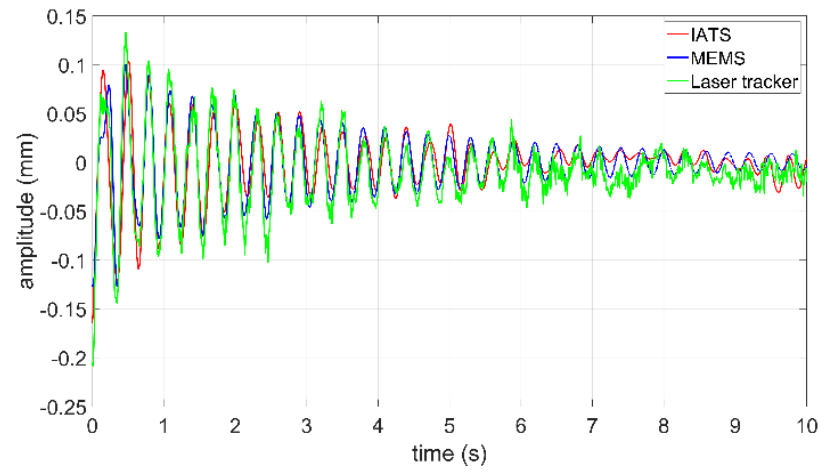

Figure 13. Overlaying of displacement time series obtained from the IATS data, the double integration of the adjusted MEMS acceleration data and the laser tracker data within the selected ambient window.

The iEKF is conducted by data fusion of the 1D displacements and $1 \mathrm{D}$ acceleration measurements with practical sampling frequencies of $10 \mathrm{~Hz}$ and $100 \mathrm{~Hz}$, respectively. The 1D displacements obtained by the IATS are considered for a 1D CUPT in the filtering step of the iEKF to minimize the displacement drift resulting from the double integration of the 1D accelerations. In order to perform iEKF, two alternative solutions were investigated. Firstly, the raw measurements from the 1D displacement and 1D acceleration datasets are included into the iEKF with different sampling frequencies by performing a multi-rate configuration similar to the work of Smyth and Wu (2006) and Ferrari et al. (2016). In this case, innovation and design matrices are adaptively updated to be compatible with the raw measurement sampling frequencies (Figure 14).

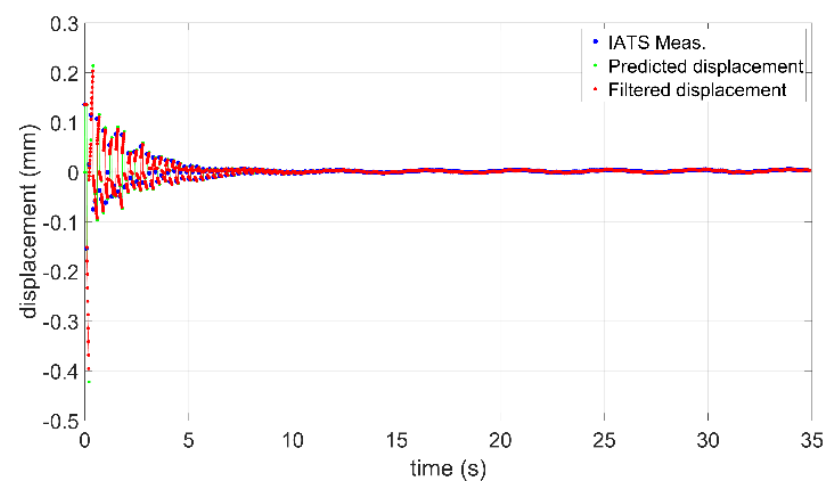

Figure 14. The displacements analysis based on iEKF and the multi-rate configuration using the displacement and acceleration measurements: the displacements obtained from the IATS (blue), the predicted displacements (green) and the filtered displacements (red).

In the second solution, the resampled displacement data from the IATS as well as the raw acceleration data are included into the iEKF. In this case, more displacement observations than the raw displacement observations are considered (Figure 15).

However, the prior knowledge about a-posteriori standard deviations of the displacement and acceleration data obtained from the estimation procedure allows to have more reliable estimation within the iEKF procedure. Consequently, such filtering procedure enables us to avoid the drift of the displacements estimates derived from the MEMS acceleration data over a longer period of time (few minutes or higher) by means of the displacement data from the IATS. In our future work, the linear drift of the offset in the DHO model can be extended to higher order polynomials to enhance the generality of the proposed algorithm.

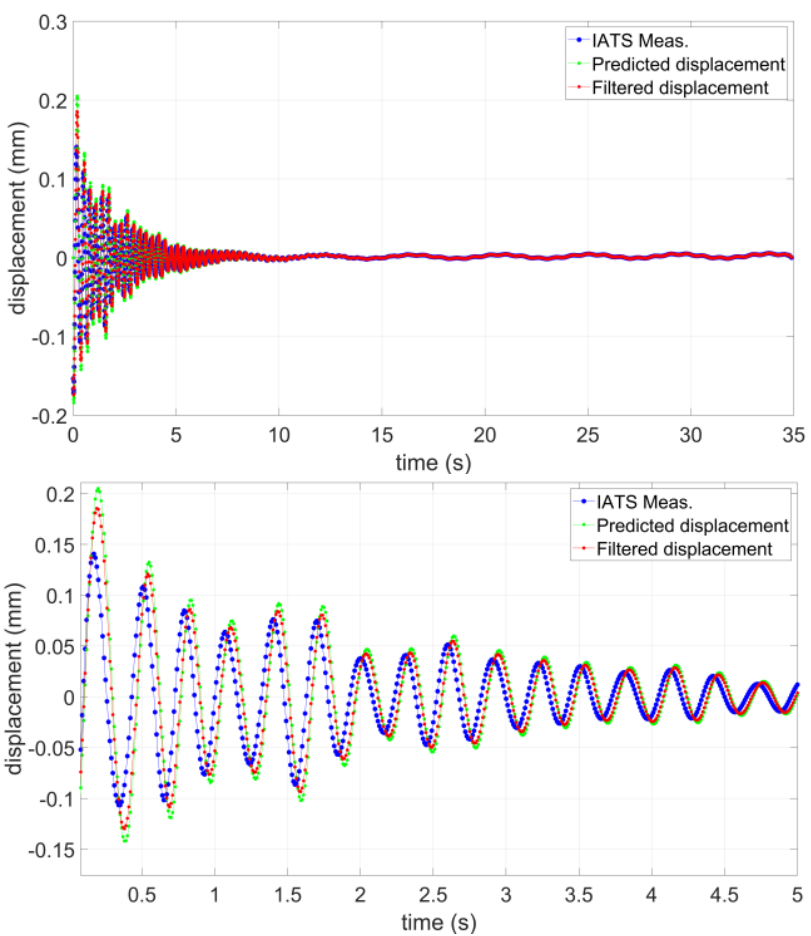

Figure 15. The displacements analysis based on iEKF and performing the resampling using the displacement and acceleration measurements (top) and its magnification (bottom): the displacements obtained from the IATS (blue), the predicted displacements (green) and the filtered displacements (red).

\section{CONCLUSIONS}

In this study the good feasibility of MEMS accelerometers supported by an IATS for short- and long-term deformation monitoring of bridge structure is shown.

To allow for a precise and reliable deformation monitoring of oscillating structures with less accuracy degradation over time, two scenarios are proposed. Firstly, a calibration procedure for MEMS accelerations is conducted to obtain calibration parameters, which enables us to later refine the measurements in a real monitoring of bridge structures. It is carried out using a KUKA youbot for fixed positions in a climate chamber and over different temperature ranges. However, the analysis reveals a minor influence of the calibration parameters on the estimated parameters for the short time interval (few minutes). The second scenario is performed to estimate the modal parameters in a controlled excitation experiment using a shaker. In addition, it enables us to observe the time synchronisation between the three used MEMS accelerometers. To this end, both scenarios support the selection process of finding the proper and optimal MEMS accelerometers.

To perform a vibration analysis, the observation model is determined based on a DHO model, an auto-correlation model in the form of an AR process as well as a stochastic model in the 
form of heavy tailed family of scaled t-distributions with an unknown degree of freedom and with unknown scale factor. The analyses prove that such a combination allows for robust, reliable and accurate estimation of the modal parameters.

The overall analysis shows that the MEMS used within the experiment are capable to identify all possible eigenfrequencies of the synthetic bridge structure with an accuracy level of submillimetre for amplitudes and much better than $0.1 \mathrm{~Hz}$ for the frequencies.

A precise and reliable double integration of the acceleration measurements is carried out for a short time (i.e. 15 - $35 \mathrm{~s}$ ) to obtain displacement changes within the selected ambient window.

To overcome a displacement drift of the MEMS accelerometers in the vertical direction, the 1D CUPT is performed based on an iEKF by involving displacement time series generated from the video frames of the IATS. The resampling of the displacement data based on their estimated modal parameters significantly improves the results.

In the future work, a possible correlation between closely spaced frequencies will be investigated. The estimation procedure for the displacement data is improved by extending the offset in the DHO model to higher order polynomials. Furthermore, a global optimization is applied to estimate the modal parameters for a longer period of time. Additionally, the influence of the calibration parameters on the estimated modal parameters within the long-term interval is investigated.

\section{ACKNOWLEDGEMENTS}

The authors would like to acknowledge the Bundesanstalt für Materialforschung und -prüfung (BAM) for providing the synthetic bridge used within this experiment. The research presented was carried out within the scope of the collaborative project 'Spatio-temporal monitoring of bridge structures using low cost sensors' with ALLSAT GmbH, which was supported by the German Federal Ministry for Economic Affairs and Energy (BMWi) and the Central Innovation Programme for SMEs (Grant ZIM Kooperationsprojekt, ZF4081803DB6).

\section{REFERENCES}

Amezquita-Sanchez, J.P. and Adeli, H, 2015. A new musicempirical wavelet transform methodology for time-frequency analysis of noisy nonlinear and non-stationary signals. Digital Signal Processing, 45, 55-68.

Bar-Shalom, Y., Li, X.-R. and Kirubarajan, T., 2001. Estimation with applications to tracking and navigation. Wiley, New York NY.

Bosch, 2016. Data sheet BNO055 Intelligent 9-axis absolute orientation sensor. Document revision 1.4. https://aebst.resource.bosch.com/media/_tech/media/datasheets/BSTBNO055-DS000.pdf (10 January 2019).

Duff, K., and Hyzak, M., 1997. Structural monitoring with GPS. https://www.fhwa.dot.gov/publications/publicroads/97spring/gp s.cfm (2 November 2018).

Ehrhart, M. and Lienhart, W., 2015a. Image-based dynamic deformation monitoring of civil engineering structures from long ranges. Proc SPIE 2015; 9405: 94050J.
Ehrhart, M. and Lienhart, W., 2015b. Monitoring of civil engineering structures using a state-of-the-art image assisted total station. Journal of applied geodesy, 9(3), 174-182.

Ferrari, R., Pioldi, F., Rizzi, E., Gentile, C., Chatzi, E. N., Serantoni, E. and Wieser, A., 2016. Fusion of wireless and noncontact technologies for the dynamic testing of a historic RC bridge. Measurement Science and Technology, 27(12), 124014.

Grompone von Gioi R, Jakubowicz J, Morel JM, Randall, G., 2012. LSD: a line segment detector. Image Process On Line, 2, $35-55$.

Hexagon Metrology, 2015. Leica absolute Tracker AT960: Product Brochure (2 July 2019).

Kaehler A and Bradski G., 2016. Learning OpenCV 3: computer vision in $\mathrm{C}++$ with the OpenCV library. Sebastopol, CA: O’Reilly Media, Inc.

Kalman, R. E., 1961. New methods and results in linear prediction and filtering theory. Research Institute for Advanced Study, Baltimore, Md., Tech. Rep. 61-1.

Kargoll, B., Omidalizarandi, M., Loth, I., Paffenholz, J. A., and Alkhatib, H., 2018. An iteratively reweighted least-squares approach to adaptive robust adjustment of parameters in linear regression models with autoregressive and $\mathrm{t}$-distributed deviations. Journal of Geodesy, 92(3), 271-297.

Kargoll, B., Omidalizarandi, M., Paffenholz, J.-A., Neumann, I., Kermarrec, G., Alkhatib, H., 2019. Bootstrap tests for model selection in robust vibration analysis of oscillating structures, In: Proceedings of the 4th Joint International Symposium on Deformation Monitoring (JISDM), Athens.

Lienhart, W., Ehrhart, M. and Grick, M., 2017. High frequent total station measurements for the monitoring of bridge vibrations. Journal of applied geodesy, 11(1), 1-8.

Neitzel, F., Niemeier, W., Weisbrich, S., and Lehmann, M., 2012. Investigation of low-cost accelerometer, terrestrial laser scanner and ground-based radar interferometer for vibration monitoring of bridges. In Proceedings of the 6th European Workshop on Structural Health Monitoring, Dresden, Germany (pp. 542-551).

Omidalizarandi, M. and Zhou, C., 2013. Positioning based on integration of multi-sensor systems using Kalman filter and least square adjustment. International Archives of the Photogrammetry, Remote Sensing and Spatial Information Sciences, $1, \mathrm{~W} 3$.

Omidalizarandi, M., Kargoll, B., Paffenholz, J.-A. and Neumann, I., 2018. Accurate vision-based displacement and vibration analysis of bridge structures by means of an image-assisted total station. Advances in Mechanical Engineering, 10(6), 1687814018780052 .

Omidalizarand, M., Paffenholz, J.-A. and Neumann I., 2019a. Automatic and accurate passive target centroid detection for applications in engineering geodesy. Survey Review, 51(367), 318-333.

Omidalizarand, M., Herrmann, R., Marx, S., Kargoll, B., Paffenholz, J.-A. and Neumann I., 2019b. A validated robust and automatic procedure for vibration analysis of bridge structures using MEMS accelerometers (Under preparation). 
Psimoulis, P. A. and Stiros, S.C., 2013. Measuring deflections of a short-span railway bridge using a robotic total station. Journal of Bridge Engineering, 18(2), 182-185.

Roberts, G. W., Meng, X., and Dodson, A. H., 2004. Integrating a global positioning system and accelerometers to monitor the deflection of bridges. Journal of Surveying Engineering, 130(2), $65-72$.

Schill, F., Eichhorn, A., 2019. Deformation Monitoring of Railway Bridges with a Profile Laser Scanner. In: zfv 144 (2), S. 109-118. DOI: 10.12902/zfv-0248-2018.

Shin, E. H. and El-Sheimy, N., 2002. A new calibration method for strapdown inertial navigation systems. Z. Vermess, 127, 110 .

Smyth, A., \& Wu, M., 2006. Multirate Kalman filtering for the data fusion of displacement and acceleration measurements. In Smart Structures and Materials 2006: Sensors and Smart Structures Technologies for Civil, Mechanical, and Aerospace Systems (Vol. 6174, p. 61741G). International Society for Optics and Photonics. 